Medienpreis der Stiftung für das behinderte Kind

\section{Prävention der Alkoholembryopathie}

Für ihren Fernsehbeitrag „Schwangerschaft und Alkohol" hat die Stiftung für das behinderte Kind die freie Journalistin für den Rundfunk Berlin-Brandenburg, Ursula Stamm, mit dem Medienpreis „Prävention in der Schwangerschaft" ausgezeichnet. Der Preis ist mit 3000 Euro dotiert.

Das fetale Alkoholsyndrom ist die häufigste nicht genetisch bedingte Ursache für geistige Behinderungen. Eines von 300 Kindern in Deutschland wird mit dem Vollbild des Alkoholsyndroms einschließlich organischer Fehlbildungen und kognitiver Behinderung geboren. Jedes Jahr kommen etwa 10000 Neugeborene mit Alkoholschäden auf die Welt. In einer Studie der Charité gaben fast $60 \%$ der befragten Schwangeren an, gelegentlich Alkohol zu trinken.

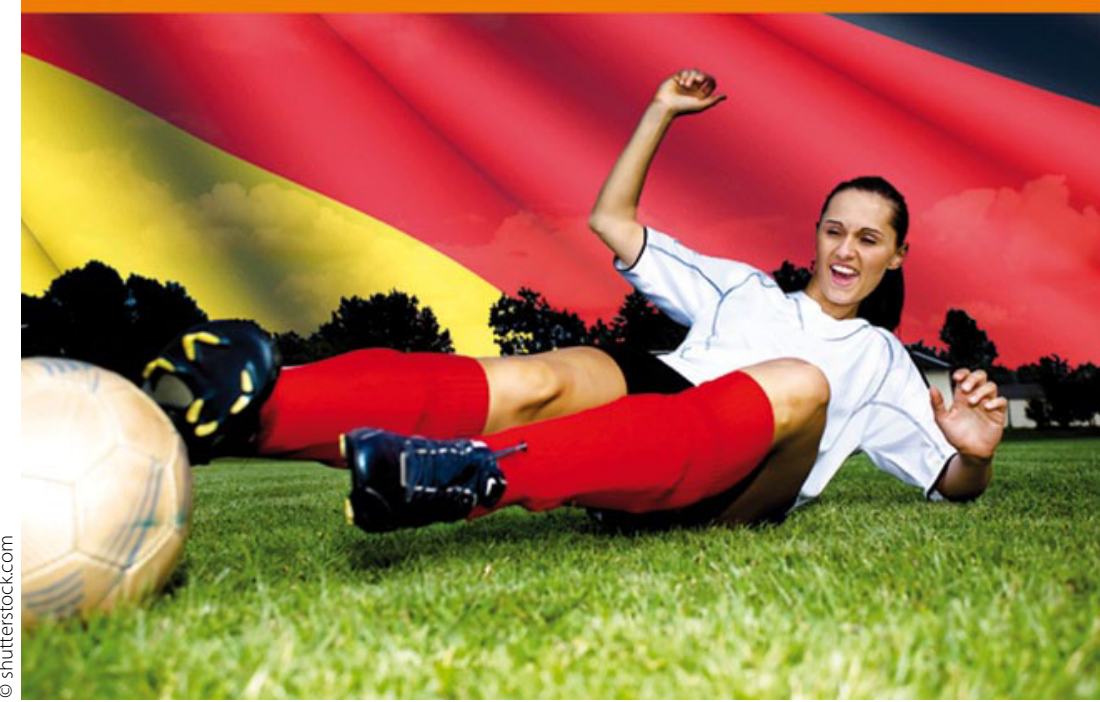

Vom 26. Juni bis zum 17. Juli kämpfen die besten Fußballerinnen der Welt um den Weltmeister-Titel 2011. Wir begleiten das "Sommermärchen“ mit unserem Frauen-WM-Quiz auf springermedizin.de. An jedem Tag des Turniers stellen wir Ihnen eine Frage rund um den Frauenfußball. Machen Sie mit und gewinnen Sie attraktive Preise.

\title{
Laufen islamische Sportlerinnen unter dem Hijab zu heiß?
}

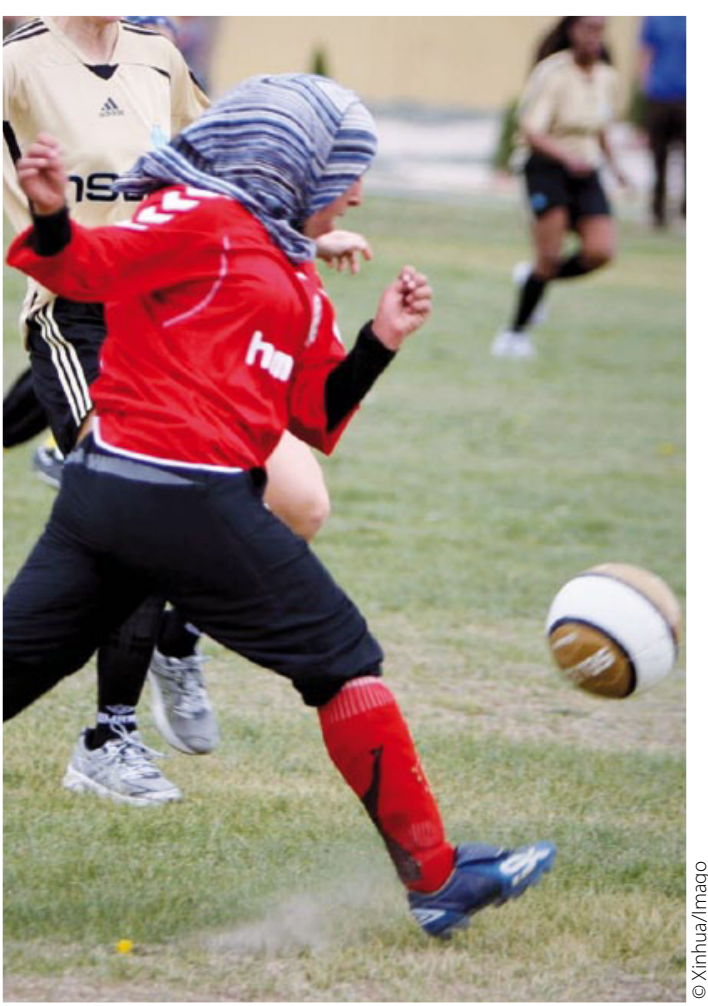

Warmes Outfit aber nicht gesundheitsgefährdend.
- Unter islamischer Sportkleidung wird es zwar heißer und es fließt mehr Schweiß, aber die Gefahr für Herz und Kreislauf der Frauen ist nicht größer als beim Training im üblichen Sporttrikot, fanden US-Wissenschaftler heraus. Hierzu schickten sie Frauen mit unterschiedlicher Bekleidung aufs Laufband.

Ein Hitzschlag ist derzeit die dritthäufigste Todesursache bei amerikanischen Athleten. Kein Wunder also, dass sich die Mediziner Sorgen um die Gesundheit verschleierter Sportlerinnen machen. Um die Belastung unter Hitzestress zu untersuchen, traten die Probandinnen bei $30{ }^{\circ} \mathrm{C}$ und 56\% Luftfeuchtigkeit sowohl im üblichen Fußballdress als auch in typisch islamischer Sportkleidung (Ganzkörperbedeckung einschließlich Kopf und Hals) an und ließen ihre physiologischen Reaktionen messen. Die Studie verglich u. a. Herzfrequenz, Flüssigkeitshaushalt und das Mikroklima zwischen der Haut und der Kleidung unter den beiden Bedingungen. Hierzu mussten die Teilnehmerinnen zwei separate Trainingsläufe von 45 Minuten absolvieren. Während der Übung und in der 40-minütigen Ruhephase wurden über einen "Micro-environment-Sensor" die physiologischen Parameter bestimmt.

Die Messungen ergaben, dass die Frauen unter der islamischen Sportkleidung während einer Laufbandübung bei $30^{\circ} \mathrm{C}$ mehr schwitzten und die Temperatur zwischen Haut und Bekleidung signifikant höher war, als wenn sie die übliche Sportbekleidung trugen $\left(33,3\right.$ vs. $32,0^{\circ} \mathrm{C}$ ). Trotzdem hatte die Art der Bekleidung keinen Einfluss auf Kerntemperatur, Hauttemperatur, Herzfrequenz, Flüssigkeitsbilanz oder den Krafteinsatz.

Nun hat die FIFA für die iranischen Fußballfrauen die Weltmeisterschaft noch vor dem Beginn abgepfiffen mit der Begründung, dass das Tragen des Hijab auf dem Feld nicht dem offiziellen FIFA-Reglement entspricht. Ein Gesundheitsproblem für diese Spielerinnen scheint nach der aktuellen Studie jedenfalls ausgeräumt.

ST =

Davis J-K et al. Fluid balance, thermal stress, and post exercise response in women's Islamic athletic clothing. Eur J Appl Physiol online 09 June 2011 